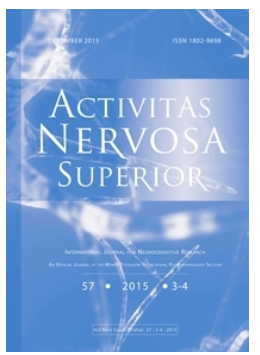

\title{
FRONTIERS IN CLINICAL DRUG RESEARCH CNS AND NEUROLOGICAL DISORDERS
}

Edited by Atta ur-Rahman, Bentham Science Publishers, 2013, 367 pages

This is an undeniably a timely book, while neurological disorders remain a therapeutic area where there is presently a scarcity of clinically approved drugs. Endorsing my opinion on the timelines of the book's theme, chapters in the book cover cutting-edge contributions of great prominence in neuroscience. In its most recent volume of the book-series, a breadth of new milestones to treat neurodegenerative disorder is covered in all of its six chapters (Chapter 1: role of ATP-sensitive $\mathrm{K}+$ channels as a novel target for tetrahydroprotoberberines' neuroprotection; Chapter 2: role of cannabinoids and their receptors in cell-based therapies for neurodegenerative disorders; Chapter 3: targeting AMPA glutamate receptor to treat neurological disorders; Chapter 4: pathogenesis of polyglutamine ataxias; Chapter 5: Use of ketogenic and atkins diets in pediatric neurology therapy; Chapter 6: role of genotoxic stress in diabetes and neurodegenerative disorders). Each chapter covers an extensive, critically evaluated body of work on the biology, pathogenesis, pharmacological evidence, targets, and mechanisms of action of active agents to treat the respective disease in a very compelling way. Complemented by a comprehensive listing of the original literature and cross-referenced indexes, I believe that this is an absolute go-to book for both experienced scientists from the drug development/discovery community and the beginners in this therapeutic area.

Reviewed by Zareen Amtul

University of Karachi 